\title{
AUECG
}

\section{Perfil dos periódicos científicos produzidos pelas instituições de ensino superior de alagoas}

\author{
Patricia Pedri \\ Universidade Federal de Alagoas \\ patriciapedri@gmail.com \\ Ronaldo Ferreira Araujo \\ Universidade Federal de Alagoas \\ ronaldfa@gmail.com
}

Como citar:

PEDRI, P.; ARAÚJO, R. F. Perfil dos periódicos científicos produzidos pelas instituições de ensino superior de alagoas. In: ABEC MEETING, 2, 2018, São Paulo. Anais... São Paulo: Associação Brasileira de Editores Científicos, 2018. p. 1-6.

http://dx.doi.org/10.21452/abecmeeting.2018.172

\section{RESUMO}

Apresenta os resultados de uma pesquisa exploratória de abordagem descritiva que teve por objetivo geral o mapeamento dos periódicos científicos mantidos por Instituições de Ensino Superior (IES) que atuam no estado de Alagoas. Os objetivos específicos compreendem a caracterização dos periódicos quanto: ao ano de criação, sistema, ISSN, área de conhecimento, periodicidade de publicação, estrato Qualis/CAPES e indexação em bases de dados. A pesquisa contribui com o debate sobre a comunicação científica e produção de periódicos, apresentando um panorama das publicações científicas do estado. O estudo indica um número comedido de revistas $(n=36)$, publicadas de 1991 a 2018 , com prevalência de periodicidade semestral (39\%). Do total de revistas 44\% não estão classificadas no Qualis/CAPES e $69 \%$ não são indexadas em bases de dados, o que inibe a visibilidade e a disseminação da informação científica publicada em Alagoas. O quadro apresentado demanda a elaboração de uma política de fomento e qualificação dos periódicos científicos do estado.

Palavras-chave: Comunicação científico. Periódicos. Publicação científica. Qualis.

\section{ABSTRACT}

It presents the results of an exploratory research with a descriptive approach that had as general objective the mapping of academic journals maintained by Higher Education Institutions that operate in the state of Alagoas, Brazil. The specific objectives include the characterization of periodicals as: year of creation, system, ISSN, area of knowledge, periodicity of publication, Qualis CAPES stratum and indexing in databases. The research contributes with the debate on the scholarly communication and production of academic journals, presenting an overview of them. The study indicates a moderate number of journals $(n=36)$, published between 1991 and 2018, with a semiannual periodicity (39\%). 44\% are not classified in Qualis/CAPES and 69\% are not indexed in databases, which inhibits the visibility and dissemination of scientific information published in Alagoas. The results presented demands the elaboration of a policy for 
the promotion and qualification of the state academic journlas.

Keywords: Scholarly communication. Academic journals. Publishing. Qualis.

\section{INTRODUÇÃO}

A essência da pesquisa científica é a produção do conhecimento, para tanto é indispensável a publicação dos resultados tendo em vista a disseminação da informação científica entre os pares e a sociedade.

Os primeiros periódicos científicos surgem no século XVII, em razão da imprescindibilidade da publicação e divulgação das descobertas científicas. O crescimento do ensino superior desperta a necessidade de publicar as produções científicas, assim "são criadas as primeiras publicações de periódicos da comunidade acadêmica" (BLATTMANN; SANTOS, 2014, p. 100).

O periódico científico é considerado o principal canal formal da comunicação científica (MEADOWS, 1999), uma vez que divulga os resultados recém-gerados e os endossa por meio de um sistema de avaliação por pares, que por sua vez atesta qualidade e credibilidade. Segundo Mueller, as funções geralmente atribuídas ao periódico científico são o estabelecimento da ciência "certificada", ou seja, a comunidade científica concedeu um aval; um canal de comunicação entre os cientistas e de divulgação mais ampla da ciência; um arquivo ou memória científica e um registro da autoria da descoberta científica (MUELLER, 1999).

Dessa forma, a presente pesquisa tem por objetivo principal o mapeamento e caracterização de periódicos mantidos por Instituições de Ensino Superior (IES) de Alagoas fornecendo elementos para um futuro debate acerca de políticas de fomento a periódicos científicos para o Estado. Ademais, promove visibilidade à produção e disseminação da informação científica de Alagoas. Os objetivos específicos do estudo foram: (a) identificar as IES que atuam no estado de Alagoas; (b) mapear os periódicos científicos mantidos pelas IES do estado de Alagoas; (c) caracterizar os periódicos levantados quanto ao ano de criação, sistema, International Standard Serial Number (ISSN), área de conhecimento, periodicidade de publicação, estrato Qualis da Coordenação de Aperfeiçoamento de Pessoal de Nível Superior (Capes) e indexação em bases de dados.

\section{METODOLOGIA}

A pesquisa está inserida no espaço científico das Ciências Sociais Aplicadas, no âmbito da Ciência da Informação e tem caráter exploratório. As técnicas aplicadas foram qualitativas e quantitativas, a primeira para o levantamento, estudo e síntese da literatura relevante ao tema, e a segunda quanto à fase empírica de levantamento, análise e descrição de dados. A revisão de literatura norteou produções técnicocientíficas como os relatórios de pesquisa, assim como a análise dos dados. $\mathrm{Na}$ fase empírica foi realizado o mapeamento dos periódicos científicos publicados em Alagoas e o levantamento de metadados dessas publicações.

O critério para a identificação das revistas consiste na presença de um editor vinculado à IES de Alagoas. A lista das IES foi obtida via Lei de Acesso à Informação (LAI) com solicitação junto ao Ministério da Educação (MEC). Após identificação das IES as fontes consultadas foram: lista de periódicos disponíveis nos websites das IES, Portal de Periódicos da Coordenação de Aperfeiçoamento de Pessoal de Nível Superior (Capes), contatos telefônicos, e-mails e visitas in loco nos programas de 
pós-graduação das IES. Essa diversidade dos meios de investigação proporcionou um levantamento mais minucioso e rigoroso dos periódicos produzidos nas instituições públicas de ensino superior de Alagoas.

Os dados levantados para a caracterização dos periódicos científicos se deu por meio de informações coletadas dos websites oficiais dos periódicos, ao passo que a atribuição do estrato Qualis da Capes foi verificado por intermédio dos dados disponíveis na Plataforma Sucupira mantida pelo Governo Federal.

\section{RESULTADOS E DISCUSSÃO}

As IES identificadas em Alagoas foram: quatro públicas e vinte e cinco privadas. Das públicas duas são federais: a Universidade Federal de Alagoas (UFAL) e o Instituto Federal de Educação, Ciência e Tecnologia de Alagoas (IFAL), e duas estaduais: a Universidade Estadual de Alagoas (UNEAL) e a Universidade Estadual de Ciências da Saúde de Alagoas (UNCISAL).

Foram levantados um total de 36 periódicos, sendo 23 mantidos pelas IES públicas, com predominância da UFAL $(20-56 \%)$ e 13 pelas privadas, com predominância do Centro Universitário Cesmac (CESMAC) (7 - 19\%), como mostra o Gráfico 1. Vale destacar que, das vinte e cinco IES privadas, apenas cinco mantém revistas científicas, em contrapartida as públicas que somam 4, somente uma não possui periódico.

Gráfico1 - Número de periódicos por IES de Alagoas.

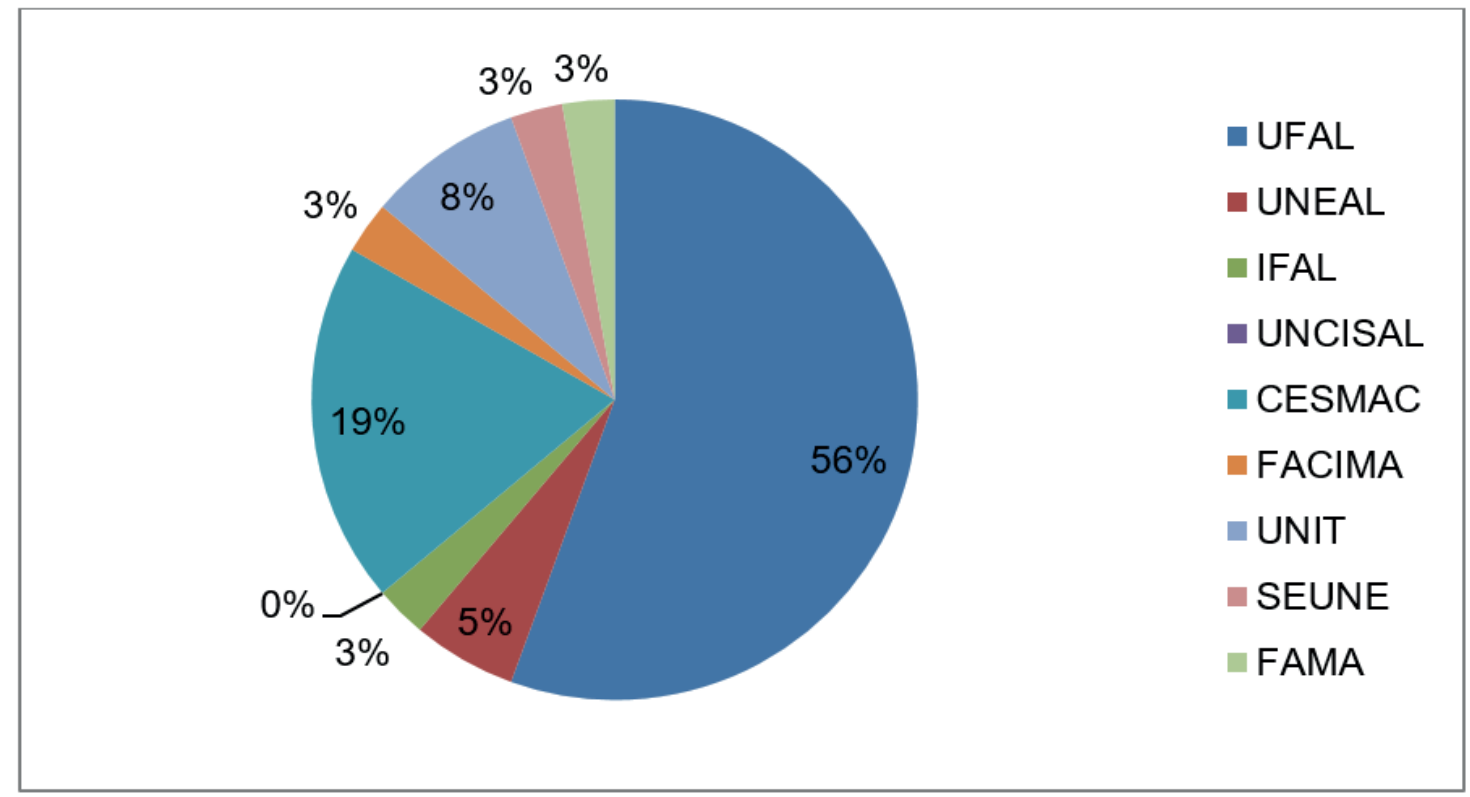

Fonte: Dados da pesquisa, 2018.

As revistas foram criadas em sua maioria no período de 2009 a 2018 (33), com exceção de duas que não informaram a data de criação, e a Revista Agrícola da UFAL que foi criada em 1991, sendo assim identificada como a revista científica mais antiga em atividade no estado. Esses dados permitem perceber, ainda que de forma morosa, o crescimento da produção de conhecimento científico em Alagoas nos últimos anos. O resultado é um pouco similar ao encontrado por Cirino et al (2017) que, ao investigarem o panorama da produção periódica de revistas científicas do Estado de Minas Gerais, identificaram uma expressiva concentração de periódicos ativos com aproximadamente 10 anos de existência (criados na década de 2000). 
No que concerne à periodicidade, a maioria das revistas estudadas são semestrais $(14-39 \%)$, seguidas das anuais $(7-19 \%)$, quadrimestrais $(6-17 \%)$ e apenas uma revista consiste em publicações trimestrais. Os dados são próximos aos encontrados por Oliveira e outros (2015) em análise às revistas mantidas pelo Portal de Periódicos da Universidade Federal de Santa Catarina (UFSC), com a periodicidade mais adotada sendo semestral, seguida da quadrimestral, com 14,3\% (6) das revistas.

Os periódicos estudados, apesar de presentes em quase todas as áreas de conhecimento, estão distribuídos de forma desigual em razão da concentração de revistas nas Ciências Humanas e nas Ciências Sociais Aplicadas com 12 publicações cada, totalizando $67 \%$ dos periódicos. A pesquisa também aponta que $25 \%$ (9) das revistas apresentam mais de uma área de conhecimento no seu escopo, ou seja, são multidisciplinares. Essa concentração em algumas áreas contrasta com a ausência de periódicos nas áreas das Ciências Biológicas e Engenharias.

De acordo com Cirino et al (2017) a forma desigual de distribuição de periódicos por área de conhecimento em um estado, no caso estudo pelos autores, o de Minas Gerais, indica a necessidade de novas pesquisas para a compreensão desse cenário e adequação das políticas de qualificação e fomento para a produção e divulgação científica no Estado.

No tocante à edição das revistas, prevaleceu o sistema Open Journal Systems (OJS) - Sistema Eletrônico de Editoração de Revistas (SEER), nome adotado pelo Instituto Brasileiro de Informação em Ciência e Tecnologia (Ibict), sendo distribuído por ele e recomendado pela Capes. Também foi constado que $92 \%$ (33) das publicações possuem ISSN, o que demonstra que os editores estão atentos à disseminação das publicações, visto que o uso do sistema OJS e a aquisição do ISSN facilitam a busca em bases catalográficas e de bibliotecas, proporcionando maior visibilidade ao periódico.

A implementação do uso da plataforma SEER por portais e revistas costuma ser justificado pelo fato de ser uma ferramenta que facilita o processo de editoração científica e também a revisão por pares. Além de disponibilizar uma série de recursos relacionados à visibilidade dos títulos. Isso a torna uma ferramenta de editoração confiável pelos editores de periódicos (BLATTMANN; ELUAN, 2009).

As universidades públicas brasileiras apresentam alto grau de aceitação do SEER, com 82,5\% (137) dos títulos que utilizam esse sistema (GARRIDO; RODRIGUES, 2010). O SEER faz parte de uma nova geração de sistemas de gerenciamento de periódicos científicos, e no Brasil surgiu como modelo alternativo de publicação do conhecimento científico com vistas a ampliar o acesso, a preservação e o impacto da busca de informação e dos resultados daí provenientes (FREIRE; SOUZA, 2010).

Quanto à qualificação, apenas vinte $(56 \%)$ periódicos apresentam estratos indicativos da qualidade Qualis da Capes, o que merece atenção por parte das IES visto que o "Qualis fornece [...] um índice que infere a qualidade das revistas, pesquisas técnicas e científicas dos programas de pós-graduação do Brasil" (OLIEVEIRA et al., 2015, p. 77). Das vinte revistas que dispõem do Qualis, onze apresentam o estrato B5, três B4, duas B3, uma B2 e duas B1 (estas últimas da área da Educação). Esse quantitativo refere-se aos indicativos de qualidade das áreas de conhecimento ascendentes das revistas. Vale lembrar que na área Multidisciplinar, que soma 9 revistas, não há possibilidade de indicar a área ascendente uma vez que são diversas. influenciando a quantidade e a qualidade de submissões de cada título, pois os títulos que melhor se classificam, pontuam mais pelos programas de pós graduação e também para a progressão funcional dos autores.

O estudo ainda demonstra que $36 \%$ (13) das revistas possuem o estrato Qualis 
em diferentes áreas do conhecimento, o que confere um aspecto multidisciplinar importante na qualificação das revistas. Entretanto, seis dessas revistas apresentam melhores avaliações em áreas diferentes da sua área original, a exemplo da Revista ReCont Registro Contábil, de Ciências Contábeis da UFAL que apresenta Qualis B3 na área de Ciências Ambientais e B5 em Ciências Contábeis. Situações como essa, demandam de atenção pois podem refletir um afastamento do foco e escopo da revista.

A indexação das revistas pesquisadas também se mostra tímida, visto que somente dez revistas (28\%) estão incluídas em alguma base de dados. O mais agravante está nas revistas mantidas pelas IES privadas, das quais nenhuma está indexada em bases de dados. Os dados são distantes dos números encontrados por Cirino et al (2017) em análise aos periódicos do Estado de Minas Gerais, onde $68 \%$ das revistas são indexadas em alguma base de dados. Vale ressaltar que revistas confiáveis e com boa indexação nas bases de dados "conseguem reunir um considerável número de artigos, logo, quanto mais relevantes forem os artigos, mais oportunidades se têm de obter uma boa visibilidade e como consequência, elevar os índices de citações das revistas" (OLIVEIRA et al, 2015, p. 86). Nesse sentido, a reduzida presença dos periódicos das IES de Alagoas nas bases de dados nacionais ou internacionais, pode inibir a acessibilidade, visibilidade e impacto das publicações científicas desses periódicos.

\section{CONSIDERAÇÕES}

Os periódicos mantidos pelas IES de Alagoas representam um quantitativo ainda acanhado se comparado com o montante de cursos oferecidos pelas IES pesquisadas, que somam 302 cursos de graduação. Essa desproporcionalidade revela uma carência de espaços para as publicações de produções científicas em Alagoas. Do mesmo modo, o número de periódicos que são classificados no Qualis da Capes e selecionados por indexadores, mostra-se comedido diante das demandas contemporâneas da comunidade científica, o que demonstra um alheamento à importância do processo de qualificação e indexação das revistas investigadas, para a produção científica no estado.

Diante desse quadro, seria pertinente a elaboração de uma política de fomento, tanto na criação quanto manutenção dos periódicos, a fim de publicar, armazenar, disseminar e divulgar a produção científica, no sentido de ampliar a visibilidade das publicações e aprimorar a qualidade da comunicação científica no estado.

\section{AGRADECIMENTO}

Os autores agradecem à Fundação de Amparo à Pesquisa do Estado de Alagoas (FAPEAL) pelo apoio financeiro por meio do Edital 04/2016 Chamada Universal, do qual a pesquisa foi contemplada com o processo: 600301057/2016.

\section{REFERÊNCIAS}

BLATTMANN, U.; SANTOS, R. N. M. dos. Revistas científicas brasileiras e sua visibilidade no acesso aberto. Informação \& Sociedade, v. 24, p. 99-106, 2014. Disponível em: <http://www.brapci.inf.br/index.php/article/view/0000018410/ cf29bdf9efdbe86710803b5bfced87f9>. Acesso em: 01 jun. 2018. 
BLATTMANN, U.; ELUAN, A. A. A cultura e a diversidade no acesso do portal periódicos da Universidade Federal de Santa Catarina. Revista AIBDA, Riverside, CA, v. 30, n. 02/01, 2009.

CIRINO, S. D. et al. Política pública de periódicos científicos para o estado de Minas Gerais (Brasil). In: Encontro Ibérico da Ciência da Informação, Coimbra, n.8, nov., 2017. Anais... EDICIC17, Coimbra: UC, 2017.

FREIRE, I. M.; SOUZA, A. P. Revista pesquisa Brasileira em Ciência da Informação e Biblioteconomia - PBCIB: um mapeamento temático da produção científica à luz da análise de conteúdo. Informação e informação, Londrina, v. 15, n. 2, p. 109127, jul./dez. 2010. Disponível em: <http://www.uel.br/revistas/uel/index.php/ informacao/article/view/5338>. Acesso em: 08 abr. 2018.

GARRIDO, I. S.; RODRIGUES, R. S. Portais de Periódicos Científicos Online:

organização institucional das publicações. Perspect. ciênc. inf., v. 15, n. 2, p. 5672, jun. 2010. Disponível em: <http://portaldeperiodicos.eci.ufmg.br/index.php/ pci/article/view/943>. Acesso em: 08 abr. 2018.

MEADOWS, A. J. A comunicação científica. Brasília: Briquet de Lemos, 1999.

MUELLER, S. P. M. O círculo vicioso que prende os periódicos nacionais. DataGramaZero, v. 0, n. 0, p. A04-0, 1999.

Disponível em: <http://www.brapci.inf.br/index.php/article/ view/0000001207/215177d99786602ac4dbdd72773633b9>. Acesso em: 09 mar. 2018.

OLIVEIRA, Aline Borges et al. Comparação entre o Qualis/Capes e os índices $\mathrm{H} \mathrm{e}$ G: o caso do portal de periódicos UFSC. Informação \& Informação, v. 20, n. 1, p. 70-91, maio 2015. Disponível em: <http://www.uel.br/revistas/uel/index.php/ informacao/article/view/17054>. Acesso em: 17 jun. 2018. 